\title{
Ovarian and endometrial endometrioid carcinomas have distinct CTNNB1 and PTEN mutation profiles
}

Melissa K McConechy ${ }^{1}$, Jiarui Ding ${ }^{2,3}$, Janine Senz ${ }^{1}$, Winnie Yang $^{1}$, Nataliya Melnyk ${ }^{1}$, Alicia A Tone ${ }^{4}$, Leah M Prentice ${ }^{1}$, Kimberly C Wiegand ${ }^{1}$, Jessica N McAlpine ${ }^{5}$, Sohrab P Shah ${ }^{2,3}$, Cheng-Han Lee ${ }^{6}$, Paul J Goodfellow ${ }^{7}$, C Blake Gilks ${ }^{6}$ and David G Huntsman ${ }^{1,2}$

${ }^{1}$ Department of Pathology and Laboratory Medicine, University of British Columbia, BC Cancer Agency, Centre for Translational and Applied Genomics, Vancouver, BC, Canada; ${ }^{2}$ Department of Molecular Oncology, BC Cancer Agency, Vancouver, BC, Canada; ${ }^{3}$ Department of Computer Science, University of British Columbia, Vancouver, BC, Canada; ${ }^{4}$ Division of Gynecology Oncology, Princess Margaret Cancer Centre, Toronto, ON, Canada; ${ }^{5}$ Division of Gynaecologic Oncology, Department of Obstetrics and Gynaecology, Vancouver General Hospital, University of British Columbia, Vancouver, BC, Canada; ${ }^{6}$ Department of Pathology and Laboratory Medicine, Vancouver General Hospital and University of British Columbia, Vancouver, BC, Canada and ${ }^{7}$ Department of Obstetrics and Gynecology, College of Medicine, Ohio State University, Columbus, OH, USA

\begin{abstract}
Ovarian endometrioid carcinomas and endometrial endometrioid carcinomas share many histological and molecular alterations. These similarities are likely due to a common endometrial epithelial precursor cell of origin, with most ovarian endometrioid carcinomas arising from endometriosis. To directly compare the mutation profiles of two morphologically similar tumor types, endometrial endometrioid carcinomas $(n=307)$ and ovarian endometrioid carcinomas $(n=33)$, we performed select exon capture sequencing on a panel of genes: ARID1A, PTEN, PIK3CA, KRAS, CTNNB1, PPP2R1A, TP53. We found that PTEN mutations are more frequent in low-grade endometrial endometrioid carcinomas $(67 \%)$ compared with low-grade ovarian endometrioid carcinomas $(17 \%)(P<0.0001)$. By contrast, CTNNB1 mutations are significantly different in low-grade ovarian endometrioid carcinomas (53\%) compared with low-grade endometrial endometrioid carcinomas (28\%) $(P<0.0057)$. This difference in CTNNB1 mutation frequency may be reflective of the distinct microenvironments; the epithelial cells lining an endometriotic cyst within the ovary are exposed to a highly oxidative environment that promotes tumorigenesis. Understanding the distinct mutation patterns found in the PI3K and Wnt pathways of ovarian and endometrial endometrioid carcinomas may provide future opportunities for stratifying patients for targeted therapeutics.

Modern Pathology (2014) 27, 128-134; doi:10.1038/modpathol.2013.107; published online 14 June 2013
\end{abstract}

Keywords: carcinoma; CTNNB1; endometrial; endometrioid; mutations; ovarian; PTEN

Ovarian and endometrial carcinomas comprise the majority of gynecological carcinomas in developed countries. ${ }^{1}$ Endometrial carcinoma is the most common with an estimated 287000 new cases

Correspondence: Dr CB Gilks, MD, FRCPC, Anatomical Pathology, JP1400, Vancouver General Hospital, 910 West 10th Avenue, Vancouver, BC V5Z 14E3, Canada or Dr DG Huntsman, MD, Department of Pathology and Laboratory Medicine, University of British Columbia, British Columbia Cancer Agency, 3427-600 West 10th Avenue, Vancouver, BC V5E 4E6, Canada. E-mail: blake.gilks@vch.ca or dhuntsma@bccancer.bc.ca Received 1 February 2013; revised 10 May 2013; accepted 11 May 2013; published online 14 June 2013 worldwide; however, ovarian carcinoma is the most lethal gynecological cancer. ${ }^{2}$ Both ovarian and endometrial carcinomas can be subclassified into the histological types: endometrioid, serous, clear cell, mixed, carcinosarcomas and mucinous carcinomas. Endometrial endometrioid carcinomas occur most frequently, accounting for $70-80 \%$ of cases; these tend to be low-grade with good prognosis and are generally cured by hysterectomy. 3,4 The remaining $20-30 \%$ of endometrial carcinomas are serous, mixed, clear cell carcinomas and carcinosarcomas. These subtypes are high-risk tumors that tend to have poor outcomes due to metastasis 
and ineffective treatment options. ${ }^{5}$ Endometrial carcinomas have been shown to possess distinct mutations specific to each subtype; endometrial endometrioid carcinomas are characterized by mutations in PTEN, ARID1A, PIK3CA, PIK3R1, CTNNB1 and KRAS (reviewed in O'Hara and Bell').

Ovarian endometrioid carcinomas account for only $10 \%$ of ovarian carcinomas. The majority of ovarian carcinomas (70-80\%) are high-grade serous carcinomas, which, similar to endometrial serous carcinomas, are aggressive with poor outcomes. The majority of ovarian endometrioid carcinomas, like endometrial endometrioid carcinomas, are also low-grade with good prognosis. ${ }^{7}$ Endometrioid carcinomas of the ovary and endometrium are both described to contain PTEN, PIK3CA, ARID1A, $P P P 2 R 1 A$, and CTNNB1 ( $\beta$-catenin) mutations; $;, 9$ however, the frequency of mutations differs in these tumor types.

There is accumulating evidence that ovarian endometrioid carcinomas arise from transformed endometriosis. ${ }^{10}$ Therefore, endometrial and ovarian endometrioid carcinomas evolve from similar precursor endometrial epithelial cells; however, the process of molecular pathogenesis is still unclear. The molecular features of ovarian endometrioid carcinomas and endometrial endometrioid carcinomas have been characterized in many studies using immunohistochemistry markers and mutational analysis by DNA sequencing (reviewed in refs 4,6,8,11). Recently, the ability to sequence multiple genes in the same cases using next-generation sequencing technology has allowed the investigation of the mutational landscapes of these tumor types. There have been studies that infer differences in mutation frequencies in ovarian endometrioid carcinomas and endometrial endometrioid carcinomas; ${ }^{12}$ however, to the best of our knowledge, no previous studies have directly compared endometrial and ovarian endometrioid mutation frequencies with a uniform technical approach in a large cohort of tumors. We recently published a study using select exon capture coupled with next-generation sequencing to determine the mutation profiles in the various subtypes of endometrial carcinoma. ${ }^{13}$ Now, we have also performed the same exon capture sequencing method and applied this to a cohort of ovarian endometrioid carcinomas. In this study, we have directly compared the mutation frequencies of seven specific genes in low-grade endometrial endometrioid carcinomas and low-grade ovarian endometrioid carcinomas.

\section{Materials and methods}

\section{Patient Samples}

We obtained frozen tumor tissue from 129 endometrial endometrioid carcinomas and 33 ovarian endometrioid carcinomas, for exon capture sequen- cing, originating from the OvCaRe Tissue Biobank repository, Vancouver, BC, Canada. Patients provided informed consent, research ethics was approved and DNA extracted as previously described. ${ }^{9}$ An additional 178 endometrial endometrioid carcinoma tumor DNA samples, with grade information available, were obtained from Washington University, St Louis, Missouri, USA. All samples from both centers have undergone histological review by gynecological pathologists. All subtype and mutational data for all endometrial carcinomas have been previously reported. ${ }^{13}$ An additional 20 ovarian endometrioid carcinoma cases (18 frozen tumors, 2 formalin-fixed paraffin embedded) were obtained from the OvCaRe Tissue Biobank repository to test CTNNB1 mutation status, so that a total of 53 ovarian endometrioid carcinomas were tested for mutation in this gene only, and 33 ovarian endometrioid carcinoma cases were tested for the other genes in the panel.

\section{Mutation Analysis}

Genomic DNA (500 ng) was used for indexed Illumina library construction, then underwent targeted enrichment using biotinylated RNA capture probes generated from cDNA clones or PCR amplicons representing exons of ARID1A, PTEN, PIK3CA, KRAS, CTNNB1, PPP2R1A, TP53, BRAF and PPP2R5C and sequenced using the Illumina (GAIIx). All sequencing validation methods and primers used are previously described. ${ }^{13}$ The two genes BRAF and PPP2R5C were excluded from subsequent analysis due to only one mutation found in BRAF and no mutations in $P P P 2 R 5 C$ in the ovarian endometrioid carcinoma cases. To validate the differences in CTNNB1 mutation frequencies, we re-sequenced the hotspot exon 3 of CTNNB1 by Sanger sequencing from all 178 low-grade endometrial endometrioid DNA obtained from Washington University. Additional Sanger sequencing validations for the hotspot exon 3 of CTNNB1 were also performed on 20 low-grade ovarian endometrioid carcinoma cases that were not included in the original select exon capture sequencing set.

\section{Bioinformatics Analysis}

Short reads were aligned to the human genome (hg18) using the BWA aligner v0.5.9. ${ }^{14}$ A Random Forest classifier trained on validated single nucleotide variants was used to remove falsepositive calls. ${ }^{15}$ Single-nucleotide variants in the Catalogue of Somatic Mutations in Cancer $^{16}$ were considered to be true positives. All analysis was performed as previously described. ${ }^{13}$

\section{Statistical Analysis}

Fisher's exact tests were used to test the significance of associations between mutations within subtypes. 
All tests were two-tailed and $P$-values $<0.05$ were considered significant. The Benjamini-Hochberg ${ }^{17}$ method was used to adjust $P$-values to account for multiple comparisons ( $\mathrm{R}$ stats package).

\section{Results}

To determine the differences in somatic mutation frequencies between endometrial and ovarian endometrioid carcinomas, we used select gene exon capture sequencing of ARID1A, PTEN, PIK3CA, KRAS, CTNNB1, PPP2R1A and TP53. This was

Table 1 Comparison of mutation frequencies in low-grade ovarian endometrioid carcinomas and low-grade endometrial endometrioid carcinomas

\begin{tabular}{lccrr}
\hline & $\begin{array}{c}\text { Low-grade } \\
\text { ovarian } \\
\text { endometrioid } \\
\text { (grades 1 } \\
\text { and 2) } \\
(\mathrm{n}=30)\end{array}$ & $\begin{array}{c}\text { Low-grade } \\
\text { endometrial } \\
\text { endometrioid } \\
\text { (grades 1 } \\
\text { and 2) } \\
(\mathrm{n}=276)\end{array}$ & $\begin{array}{c}\text { Fisher's } \\
\text { exact test } \\
\text { (P-value) }\end{array}$ & $\begin{array}{c}\text { Adjusted } \\
\text { P-value }\end{array}$ \\
\hline PTEN & $5(17 \%)$ & $185(67 \%)$ & $\mathbf{1 . 0 8 e - 0 7}$ & $\mathbf{0 . 0 0 1}$ \\
PIK3CA & $12(40 \%)$ & $107(39 \%)^{\mathrm{a}}$ & 1 & 1 \\
ARID1A & $9(30 \%)$ & $129(47 \%)$ & 0.086 & 0.120 \\
KRAS & $10(33 \%)$ & $50(18 \%)^{\mathrm{a}}$ & 0.055 & 0.120 \\
CTNNB1 & $16(53 \%)$ & $76(28 \%)^{\mathrm{a}}$ & $\mathbf{0 . 0 0 6}$ & $\mathbf{0 . 0 2 0}$ \\
PPP2R1A & $5(17 \%)$ & $19(7 \%)$ & 0.071 & 0.120 \\
TP53 & $2(7 \%)$ & $28(10 \%)$ & 1 & 1 \\
\hline & & & &
\end{tabular}

${ }^{*} P$-values are adjusted using the Benjamini-Hochberg method.

Values in bold are statistically significant.

${ }^{a}$ Additional mutations have been verified by Sanger sequencing post original publication. performed using 33 cases of ovarian endometrioid and 307 cases of endometrial endometrioid carcinomas (Supplementary Table S1). Comparison of the mutational frequencies of low-grade (grades 1 and 2) endometrial endometrioid carcinomas to low-grade (grades 1 and 2) ovarian endometrioid carcinomas showed a significant difference for PTEN (adjusted $P<0.0007)$ and CTNNB1 (adjusted $P=0.02$ ) mutations (Table 1, Figure 1). PTEN mutations were found in $67 \%$ of low-grade endometrial endometrioid carcinomas, whereas $17 \%$ of low-grade ovarian endometrioid carcinomas harbor PTEN mutations. Mutations of CTNNB1 were identified in $53 \%$ of ovarian endometrioid carcinomas and in only $28 \%$ of endometrial endometrioid carcinomas. This frequency of CTNNB1 mutations in endometrial endometrioid carcinomas is slightly higher than previously published, ${ }^{13}$ due to additional validations of mutations using Sanger sequencing and select exon capture sequencing. To further verify the high CTNNB1 mutation frequency found in ovarian endometrioid carcinomas, we acquired an additional 20 low-grade ovarian endometrioid carcinomas cases and tested for hotspot CTNNB1 mutations using direct Sanger sequencing. We found that $45 \%$ (9 of 20) of these cases contained hotspot CTNNB1 mutations, bringing the overall frequency to $50 \%$ (25 of 50 ) CTNNB1 mutations in low-grade ovarian endometrioid carcinomas (Supplementary Table S1). The mutation frequencies of PIK3CA, ARID1A, PPP2R1A and TP53 are not significantly different between low-grade endometrial endometrioid carcinomas and ovarian endometrioid carcinomas. There is a trend towards more KRAS mutations in low-grade ovarian endometrioid
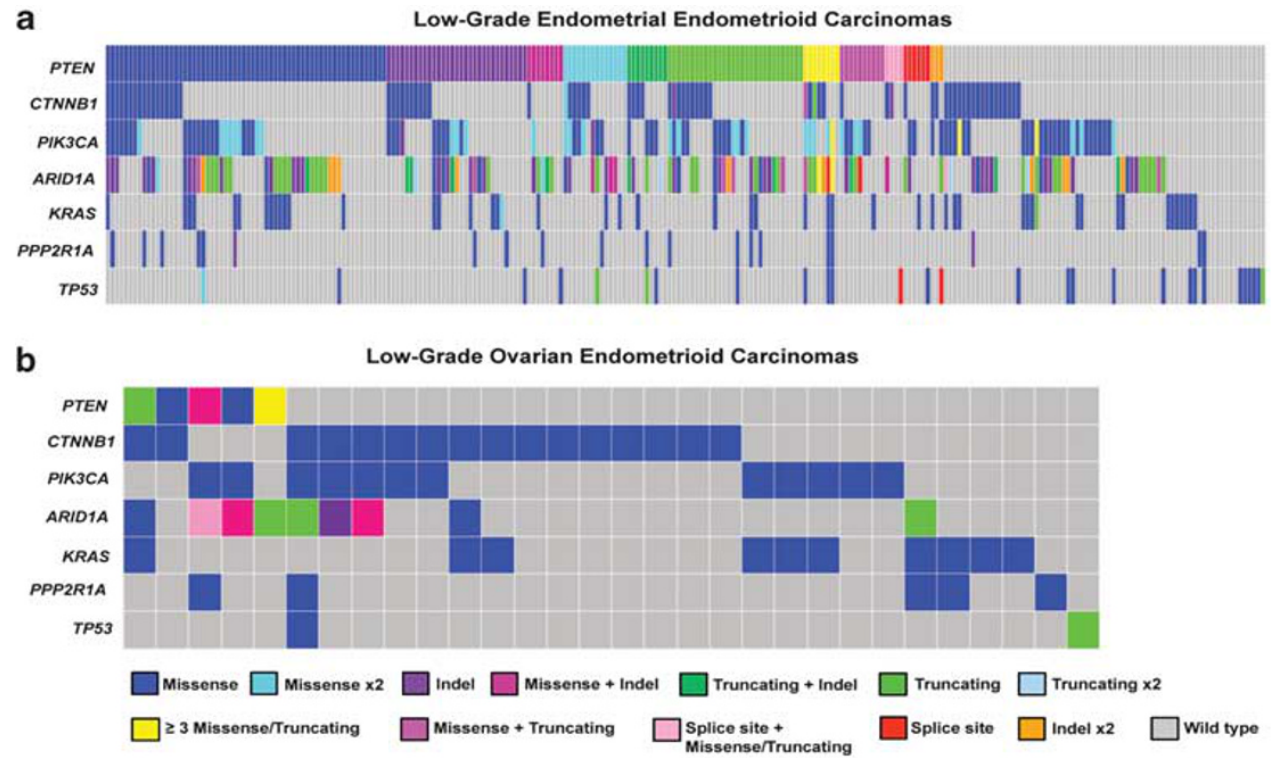

Figure 1 Low-grade ovarian and endometrial endometrioid mutation profiles. (a) Low-grade endometrial endometrioid carcinomas, including grades 1 and $2(n=276)$. (b) Low-grade ovarian endometrioid carcinomas, including grades 1 and 2 ( $n=30$ ). Individual columns designate one tumor case, and rows indicate genes. All colored boxes specify a genetic alteration such as missense, truncating, indels, splice site mutations and combinations of these mutations. Grey boxes indicate no mutations were identified by sequencing. These colors are specifically shown in the color legend. 
Table 2 Comparison of the mutation frequencies of high-grade ovarian endometrioid carcinomas and high-grade endometrial endometrioid carcinomas

\begin{tabular}{lcc}
\hline & $\begin{array}{c}\text { High-grade } \\
\text { ovarian endometrioid } \\
\text { (grade 3) }(\mathrm{n}=3)\end{array}$ & $\begin{array}{c}\text { High-grade endometrial } \\
\text { endometrioid } \\
\text { (grade 3) }(\mathrm{n}=31)\end{array}$ \\
\hline PTEN & $1(33 \%)$ & $27(87 \%)$ \\
PIK3CA & $1(33 \%)$ & $17(55 \%)$ \\
ARID1A & $0(0 \%)$ & $18(58 \%)$ \\
KRAS & $1(33 \%)$ & $8(26 \%)$ \\
CTNNB1 & $1(33 \%)$ & $6(19 \%)$ \\
PPP2R1A & $0(0 \%)$ & $4(13 \%)$ \\
TP53 & $1(33 \%)$ & $10(32 \%)$ \\
\hline
\end{tabular}

carcinomas $(33 \%)$ compared with low-grade endometrial endometrioid carcinomas (18\%); however, this is not significant after multiple comparison adjustments (Table 1 ).

The comparisons of high-grade (grade 3) endometrial endometrioid carcinomas with high-grade ovarian endometrioid carcinomas are limited by the rarity of high-grade ovarian endometrioid carcinomas $(n=3)$. Each high-grade ovarian endometrioid carcinomas case harbors different mutations in different genes. Similarly to low-grade endometrial endometrioid carcinomas, high-grade endometrial endometrioid carcinomas also have a high frequency of PTEN mutations (87\%) and a lower frequency of CTNNB1 mutations (19\%) (Table 2).

Most CTNNB1 mutations found in both ovarian endometrioid carcinoma and endometrial endometrioid carcinoma involve known phospho-acceptor sites. In ovarian endometrioid carcinomas, 16 of $25(64 \%)$ contain CTNNB1 mutations that affect serine (S33 and S37) or threonine (T41) amino-acid residues, which are phosphorylation targets for glycogen synthase kinase 3-beta (GSK3 $\beta$ ). Similarly in low-grade endometrial endometrioid carcinomas, 43 of $76(57 \%)$ of the CTNNB1 mutations are located at serine (S33, S37, S45) and threonine (T41) residues (Supplementary Table S1). Our analysis of all CTNNB1 coding sequences also revealed additional somatic mutations outside the hotspot serine/threonine residues.

\section{Discussion}

The distinct molecular abnormalities of endometrial and ovarian carcinoma subtypes will be the basis for subtype specific treatment and may become an essential component of stratified management strategies. Standard treatment options for ovarian and endometrial carcinomas have not yet changed, but a shift towards subtype-specific clinical trials highlights the need to better understand the molecular abnormalities and potential therapeutic targets in the different subtypes. ${ }^{18}$ The same subtypes at different sites in the gynecological tract, endometrial endometrioid carcinomas and ovarian endometrioid carcinomas, have indistinguishable morphology, clinical similarities and share at least some genetic abnormalities. We have directly compared the mutation frequencies in the same gene set, using the same technology, in low-grade ovarian and endometrial endometrioid carcinomas. This has shown that there are not only similar mutation patterns but also two distinct differences.

Previous literature often refers to endometrial and ovarian endometrioid carcinomas as having similar molecular alterations. ${ }^{19,20}$ The same genes are often mutated; however, some suggest that ovarian endometrioid carcinomas and endometrial endometrioid carcinomas have similar frequencies of CTNNB1 $^{12}$ and PTEN mutations ${ }^{21}$ but do not directly compare the two tumor types in the same study. In this study, PTEN mutations are found at higher frequencies in low-grade endometrial endometrioid carcinomas compared with low-grade ovarian endometrioid carcinomas, and CTNNB1 mutations at higher frequencies in ovarian endometrioid carcinomas compared with endometrial endometrioid carcinomas. One limitation of this study is the small number of lowgrade ovarian endometrioid carcinomas $(n=30)$ compared with the number of low-grade endometrial endometrioid carcinomas $(n=276)$, in part, a reflection on the relative rarity of ovarian endometrioid carcinomas. Previous studies have reported low-grade ovarian endometrioid carcinomas with CTNNB1 mutation frequencies between 16 and $54 \%,{ }^{22-28}$ with an average frequency of $40-50 \%$. This range of CTNNB1 mutation frequency is due to varying methods of detection and limited exon sequencing. PTEN mutations are reported in only about $20 \%$ of ovarian endometrioid carcinomas. ${ }^{29,30}$ All these studies also assessed small cohorts of ovarian endometrioid carcinomas, and reported similar mutation frequencies for both CTNNB1 and PTEN.

Recently, our group $^{13}$ and others ${ }^{31-33}$ have reported mutation frequencies in a large number of endometrial endometrioid carcinomas. Byron et a $1^{32}$ report a 19\% CTNNB1 mutation frequency from 466 endometrial endometrioid tumors. In this study, we report a slightly higher CTNNB1 mutation frequency of $28 \%$ from 276 low-grade endometrial endometrioid carcinomas. This difference is most probably due to differences in sequencing (hotspot vs all exons) and analysis methods used. The current study included 25 endometrial endometrioid tumor samples also analyzed by Byron et al. ${ }^{32}$ There were 66 CTNNB1 and 46 KRAS mutations reported in the original paper, ${ }^{13}$ whereas we have now identified 76 CTNNB1 and 50 KRAS mutations, all validated by Sanger sequencing, indicating a low false-negative rate in the earlier study. This does not change the conclusions of that study ${ }^{13}$ but does indicate that there was a slight underestimation of mutation frequencies. In 
conclusion, our study confirms observations from other studies suggesting that there are differences in PTEN and CTNNB1 mutation rates in ovarian endometrioid carcinomas and endometrial endometrioid carcinomas. Similarly, ovarian and endometrial serous carcinoma subtypes are morphologically equivalent and were often thought to have similar mutation patterns, with both showing a high frequency of TP53 mutations. However, more detailed sequencing analyses of these tumor types have revealed mutational profile differences. Mutations of PPP2R1A are found at high frequencies in endometrial serous carcinomas but are rarely found in ovarian high-grade serous carcinomas. ${ }^{9,34}$ More recent studies have identified high frequencies of other gene mutations (ie, FBXW7, CHD4), ${ }^{35,36}$ in endometrial serous carcinomas but not in ovarian serous carcinomas. ${ }^{37}$

The majority of ovarian endometrioid carcinomas are believed to arise from endometriosis. ${ }^{8,38}$ Although ovarian and endometrial endometrioid carcinomas may develop from the same cell type, namely the endometrial epithelial cell, these two tumor types are exposed to different microenvironments that may reflect differences in their mutation spectrums. Endometrial endometrioid carcinomas frequently occur in postmenopausal women with unopposed estrogen; ${ }^{39}$ exposure to high estrogen and low progesterone levels have been found to increase proliferation of endometrial cells, thus increasing the risk of tumorigenesis. ${ }^{40}$ Chromosomal aberrations have been identified at a high frequency in ovarian endometriotic cysts compared with extra-gonadal endometriosis. ${ }^{41}$ Endometriosis is thought to occur via retrograde menstruation, where endometrial epithelial cells travel from the uterus through the fallopian tubes and can establish as an endometriotic cyst within the ovary. ${ }^{42}$ This creates a unique microenvironment where menstruationlike blood and necrotic tissue is trapped within the cyst, resulting in high concentrations of iron in a confined space, ${ }^{43}$ causing oxidative stress and a hypoxic environment leading to DNA damage and mutation accumulation. ${ }^{44,45}$ The mutational analysis of a small number of endometriosis lesions has mostly been confined to a subset of genes, PTEN, CTNNB1 and KRAS, with only a small number of somatic mutations found in $P T E N^{29}$ and KRAS. ${ }^{46}$ It will be important to determine the malignant transformation pathways of endometriosis to ovarian endometrioid carcinomas and, in so doing, identify the genetic differences between the precursors of endometrial and ovarian endometrioid carcinomas.

Based on the mutation frequencies found in this study, CTNNB1 mutations in the ovary and PTEN mutations in the endometrium are characteristic features of these diseases. Mutations in CTNNB1 and deregulation of the Wnt pathway are well-established pathways in cancer signaling first characterized in colorectal cancers. ${ }^{47,48}$ As seen in our study, the majority of CTNNB1 mutations change the phospho-serine/threonine sites, which affects the ability of GSK3 $\beta$ to phosphorylate $\beta$-catenin and signal degradation. Lack of $\beta$-catenin phosphorylation results in nuclear accumulation causing expression of cell proliferation and inflammatory genes (reviewed in Klaus and Birchmeier $^{49}$ ) (Figure 2). Ovarian carcinomas with an accumulation of nuclear $\beta$-catenin, either due to CTNNB1 mutations or deregulation of other Wnt family member like APC or Axin, is an indicator of good prognosis. ${ }^{23}$ Similar to the Wnt pathway, PI3K signaling is also one of the most commonly altered cancer pathways. ${ }^{50}$ PTEN acts as a negative regulator of the PI3K pathway by dephosphory lating the signaling lipid molecule PIP3 to PIP2, where PIK3CA (p110 $\alpha$ ) together with PIK3R1 (p85 $\alpha$ ) acts to phosphorylate PIP2 to PIP3, thus allowing signaling to proceed through AKT and mTOR. Mutations in both PTEN and PIK3CA can act to maintain constitutively activated PI3K signaling (reviewed in Cully et al ${ }^{51}$ ). This activation leads to the degradation of GSK $3 \beta$ and thus allows $\beta$-catenin nuclear translocation. ${ }^{52}$ The PI3K and Wnt pathways do not occur linearly, and are interactive within their own signal transduction networks as well as with other pathways, which is shown by convergence on GSK3 $\beta$ (Figure 2). There are, however, many other gene-regulation events resulting through the activation of PI3K/AKT/mTOR pathway that are not activated through the Wnt pathway. Therefore, in ovarian endometrioid carcinomas that do not respond to standard treatments, it may be beneficial to target both the Wnt pathway, to inhibit $\beta$-catenin and the PI3K pathway when PI3KCA is mutated. In the case of endometrial endometrioid

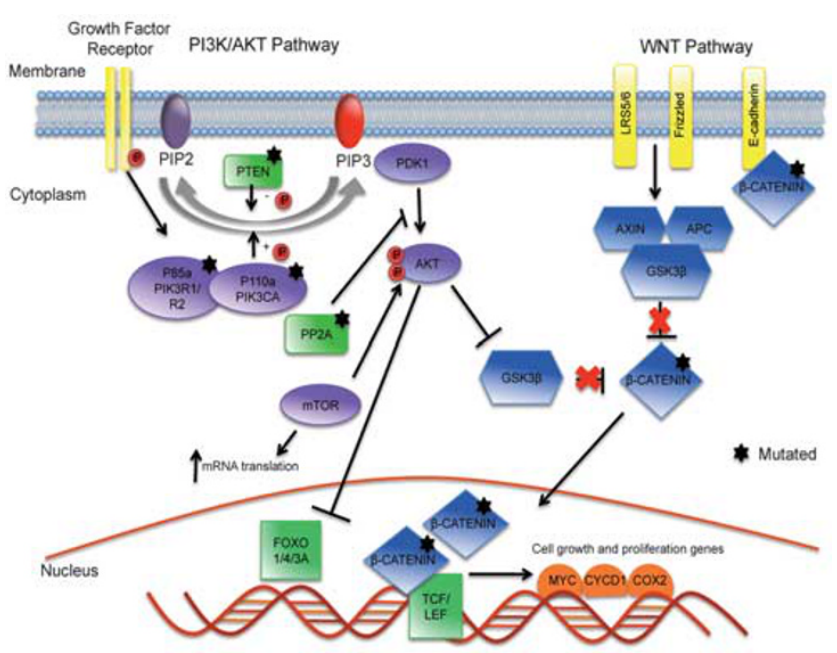

Figure 2 PI3K/AKT and WNT signaling pathways. These two signaling pathways show convergence on GSK3 $\beta$ and $\beta$-catenin. Genetic alterations caused by mutations in both pathways can result in the transcription of cell growth and proliferation genes. Mutations are indicated by black stars and are found in both ovarian and endometrial endometrioid tumors. 
carcinomas, PTEN and PIK3CA are both frequently mutated, likely leading to the upregulation of PI3K signaling, thus targeting the PI3K pathway may be of benefit. Additionally, there is no mutual exclusivity of CTNNB1, PTEN or PIK3CA mutations in endometrial endometrioid carcinomas and ovarian endometrioid carcinomas (Figure 1), indicating that they are not functionally equivalent; therefore when both pathways are mutated, simultaneously targeting the PI3K and Wnt pathways may be appropriate. Careful consideration will be needed when deciding which molecules to target in one or multiple pathways, as well as the specific cellular context.

Ovarian and endometrial endometrioid carcinomas share obvious histogenic connections and are morphologically similar; however, there are genomic differences, as shown by CTNNB1 and PTEN mutation frequencies. The occurrence of these mutations may reflect different environmental niches during oncogenesis and ultimately point toward different routes of distinct targeted therapeutics.

\section{Acknowledgements}

We would like to thank all the women who generously donated the samples used in this study. This work is supported by grants from the British Columbia (BC) Cancer Foundation, the Vancouver General Hospital (VGH)-University of British Columbia Hospital Foundation (to the OvCaRe ovarian cancer research team, Vancouver), and the Canadian Institutes of Health Research (CIHR). Work supported at Washington University, in part, by RO1CA71754 and P50CA134254 to PJG. We would also like to thank the Sequence Production and LIMS groups at Canada's Michael Smith Genome Sciences Centre for technical assistance and the fellows of the University of British Columbia Gynaecologic Oncology Program for obtaining consent from patients for data in the OvCaRe tumor bank.

\section{Disclosure/conflict of interest}

The authors declare no conflict of interest.

\section{References}

1 Jemal A, Bray F, Center MM, et al. Global cancer statistics. CA Cancer J Clin 2011;61:69-90.

2 Ferlay J, Shin HR, Bray F, et al. GLOBOCAN 2008 v1.2, Cancer Incidence and Mortality Worldwide IARC CancerBase No. 10. International Agency for Research on Cancer: Lyon, France, 2010; http://globocan.iarc.fr.

3 Catasus L, Gallardo A, Prat J. Molecular genetics of endometrial carcinoma. Diagn Histopathol 2009;15: $554-563$.

4 Samarnthai N, Hall K, Yeh IT. Molecular profiling of endometrial malignancies. Obstet Gynecol Int 2010;2010:162363.
5 Prat J. Prognostic parameters of endometrial carcinoma. Hum Pathol 2004;35:649-662.

6 O'Hara AJ, Bell DW. The genomics and genetics of endometrial cancer. Adv Genomics Genet 2012;2: 33-47.

7 Chen S, Leitao MM, Tornos C, et al. Invasion patterns in stage I endometrioid and mucinous ovarian carcinomas: a clinicopathologic analysis emphasizing favorable outcomes in carcinomas without destructive stromal invasion and the occasional malignant course of carcinomas with limited destructive stromal invasion. Mod Pathol 2005;18:903-911.

8 Kurman RJ, Shih IeM. Molecular pathogenesis and extraovarian origin of epithelial ovarian cancershifting the paradigm. Hum Pathol 2011;42:918-931.

9 McConechy MK, Anglesio MS, Kalloger SE, et al. Subtype-specific mutation of PPP2R1A in endometrial and ovarian carcinomas. J Pathol 2011;223:567-573.

10 Prowse AH, Manek S, Varma R, et al. Molecular genetic evidence that endometriosis is a precursor of ovarian cancer. Int J Cancer 2006;119:556-562.

11 Bell DA. Origins and molecular pathology of ovarian cancer. Mod Pathol 2005;18(Suppl 2):S19-S32.

12 Catasus L, Bussaglia E, Rodrguez I, et al. Molecular genetic alterations in endometrioid carcinomas of the ovary: similar frequency of beta-catenin abnormalities but lower rate of microsatellite instability and PTEN alterations than in uterine endometrioid carcinomas. Hum Pathol 2004;35:1360-1368.

13 McConechy $\mathrm{MK}$, Ding J, Cheang $\mathrm{MC}$, et al. Use of mutation profiles to refine the classification of endometrial carcinomas. J Pathol 2012;228:20-30.

$14 \mathrm{Li} \mathrm{H}$, Durbin R. Fast and accurate short read alignment with Burrows-Wheeler transform. Bioinformatics 2009;25:1754-1760.

15 Ding J, Bashashati A, Roth A, et al. Feature-based classifiers for somatic mutation detection in tumournormal paired sequencing data. Bioinformatics 2012; 28:167-175.

16 Forbes SA, Bindal N, Bamford S, et al. COSMIC: mining complete cancer genomes in the Catalogue of Somatic Mutations in Cancer. Nucleic Acids Res 2011;39:D945-D950.

17 Benjamini Y, Hochberg Y. Controlling the flse discovery rate-a practical and powerful approach to multiple testing. J Roy Stat Soc B Met 1995;57: 289-300.

18 Stuart GCE, Kitchener H, Bacon M, et al. 2010 Gynecologic Cancer InterGroup (GCIG) Consensus Statement on Clinical Trials in Ovarian Cancer Report from the Fourth Ovarian Cancer Consensus Conference. Int J Gynecol Cancer 2011;21:750-755.

19 Geyer JT, Lopez-Garcia MA, Sanchez-Estevez C, et al. Pathogenetic pathways in ovarian endometrioid adenocarcinoma: a molecular study of 29 cases. Am J Surg Pathol 2009;33:1157-1163.

20 McCluggage WG. Morphological subtypes of ovarian carcinoma: a review with emphasis on new developments and pathogenesis. Pathology 2011;43:420-432.

21 Shedden KA, Kshirsagar MP, Schwartz DR, et al. Histologic type, organ of origin, and Wnt pathway status: effect on gene expression in ovarian and uterine carcinomas. Clin Cancer Res 2005;11: 2123-2131.

22 Palacios J, Gamallo C. Mutations in the beta-catenin gene (CTNNB1) in endometrioid ovarian carcinomas. Cancer Res 1998;58:1344-1347. 
23 Gamallo C, Palacios J, Moreno G, et al. Beta-catenin expression pattern in stage I and II ovarian carcinomas: relationship with beta-catenin gene mutations, clinicopathological features, and clinical outcome. Am J Pathol 1999;155:527-536.

24 Wu R, Zhai Y, Fearon ER, et al. Diverse mechanisms of beta-catenin deregulation in ovarian endometrioid adenocarcinomas. Cancer Res 2001;61:8247-8255.

25 Wright $\mathrm{K}$, Wilson $\mathrm{P}$, Morland S, et al. Beta-catenin mutation and expression analysis in ovarian cancer: exon 3 mutations and nuclear translocation in $16 \%$ of endometrioid tumours. Int J Cancer 1999;82:625-629.

26 Saegusa M, Okayasu I. Frequent nuclear beta-catenin accumulation and associated mutations in endometrioid-type endometrial and ovarian carcinomas with squamous differentiation. J Pathol 2001;194: 59-67.

27 Sagae S, Kobayashi K, Nishioka Y, et al. Mutational analysis of beta-catenin gene in Japanese ovarian carcinomas: frequent mutations in endometrioid carcinomas. Jpn J Cancer Res 1999;90:510-515.

28 Moreno-Bueno G, Gamallo C, Perez-Gallego L, et al. Beta-Catenin expression pattern, beta-catenin gene mutations, and microsatellite instability in endometrioid ovarian carcinomas and synchronous endometrial carcinomas. Diagn Mol Pathol 2001;10: 116-122.

29 Sato N, Tsunoda H, Nishida M, et al. Loss of heterozygosity on 10q23.3 and mutation of the tumor suppressor gene PTEN in benign endometrial cyst of the ovary: possible sequence progression from benign endometrial cyst to endometrioid carcinoma and clear cell carcinoma of the ovary. Cancer Res 2000; 60:7052-7056.

30 Obata K, Morland SJ, Watson RH, et al. Frequent PTEN/MMAC mutations in endometrioid but not serous or mucinous epithelial ovarian tumors. Cancer Res 1998;58:2095-2097.

31 Cheung LW, Hennessy BT, Li J, et al. High frequency of PIK3R1 and PIK3R2 mutations in endometrial cancer elucidates a novel mechanism for regulation of PTEN protein stability. Cancer Discov 2011;1:170-185.

32 Byron SA, Gartside M, Powell MA, et al. FGFR2 point mutations in 466 endometrioid endometrial tumors: relationship with MSI, KRAS, PIK3CA, CTNNB1 mutations and clinicopathological features. PLoS One 2012;7:e30801.

33 Urick ME, Rudd ML, Godwin AK, et al. PIK3R1 (p85\{alpha\}) is somatically mutated at high frequency in primary endometrial cancer. Cancer Res 2011;71:4061-4067.

34 Shih IeM, Panuganti PK, Kuo KT, et al. Somatic mutations of PPP2R1A in ovarian and uterine carcinomas. Am J Pathol 2011;178:1442-1447.

35 Kuhn E, Wu RC, Guan B, et al. Identification of molecular pathway aberrations in uterine serous carcinoma by genome-wide analyses. J Natl Cancer Inst 2012;104:1503-1513.

36 Gallo ML, O’Hara AJ, Rudd ML, et al. Exome sequencing of serous endometrial tumors identifies recurrent somatic mutations in chromatin-remodeling and ubiquitin ligase complex genes. Nat Genet 2012;44:1310-1315.

37 Cancer Genome Atlas Research Network. Integrated genomic analyses of ovarian carcinoma. Nature 2011;474:609-615.

38 Jiang X, Morland SJ, Hitchcock A, et al. Allelotyping of endometriosis with adjacent ovarian carcinoma reveals evidence of a common lineage. Cancer Res 1998; 5:1707-1712.

39 Siiteri PK, Schwarz BE, MacDonald PC. Estrogen receptors and the estrone hypothesis in relation to endometrial and breast cancer. Gynecol Oncol 1974;2:228-238.

40 Key TJA, Pike MC. The dose-effect relationship between unopposed estrogens and endometrial mitotic rate-its central role in explaining and predicting endometrial cancer risk. Brit J Cancer 1988;57: 205-212.

41 Korner M, Burckhardt E, Mazzucchelli L. Higher frequency of chromosomal aberrations in ovarian endometriosis compared to extragonadal endometriosis: a possible link to endometrioid adenocarcinoma. Mod Pathol 2006;19:1615-1623.

42 Giudice LC. Endometriosis. N Engl J Med 2010; 362:2389-2398.

43 Yamaguchi K, Mandai M, Toyokuni S, et al. Contents of endometriotic cysts, especially the high concentration of free iron, are a possible cause of carcinogenesis in the cysts through the iron-induced persistent oxidative stress. Clin Cancer Res 2008;14:32-40.

44 Van Langendonckt A, Casanas-Roux F, Dolmans MM, et al. Potential involvement of hemoglobin and heme in the pathogenesis of peritoneal endometriosis. Fertil Steril 2002;77:561-570.

45 Kobayashi H, Kajiwara H, Kanayama S, et al. Molecular pathogenesis of endometriosis-associated clear cell carcinoma of the ovary (Review). Oncol Rep 2009;22:233-240.

46 Vestergaard AL, Thorup K, Knudsen UB, et al. Oncogenic events associated with endometrial and ovarian cancers are rare in endometriosis. Mol Hum Reprod 2011;17:758-761.

$47 \mathrm{Su}$ LK, Vogelstein B, Kinzler KW. Association of the APC tumor suppressor protein with catenins. Science 1993;262:1734-1737.

48 Rubinfeld B, Souza B, Albert I, et al. Association of the APC gene product with beta-catenin. Science 1993;262:1731-1734.

49 Klaus A, Birchmeier W. Wnt signalling and its impact on development and cancer. Nat Rev Cancer 2008; 8:387-398.

50 Samuels Y, Wang Z, Bardelli A, et al. High frequency of mutations of the PIK3CA gene in human cancers. Science 2004;304:554.

51 Cully M, You H, Levine AJ, et al. Beyond PTEN mutations: the PI3K pathway as an integrator of multiple inputs during tumorigenesis. Nat Rev Cancer 2006;6:184-192.

52 McCubrey JA, Steelman LS, Chappell WH, et al. Mutations and deregulation of Ras/Raf/MEK/ERK and PI3K/PTEN/Akt/mTOR cascades. Oncotarget 2012; 3:954-987.

Supplementary Information accompanies the paper on Modern Pathology website (http://www.nature.com/ modpathol) 